\title{
An Experimental Framework for Testing Web Prefetching Techniques
}

\author{
Josep Domènech, Ana Pont, Julio Sahuquillo, and José A. Gil \\ Department of Computer Engineering \\ Polytechnic University of Valencia \\ Valencia, Spain \\ jodode@doctor.upv.es, \{apont,jsahuqui,jagil\}@disca.upv.es
}

\begin{abstract}
The popularity of web objects, and by extension the popularity of the web sites, besides the appearance of clear footprints in user's accesses that show a considerable spatial locality, make possible to predict future accesses based on the current ones. This fact permits to implement also prefetching techniques in Web Architecture in order to reduce the latency perceived by the users. Although the open literature presents some approaches in this sense, the huge variety of prefetching algorithms, and the different scenarios and conditions where they are applied make very difficult to compare performance and to obtain correct conclusions that permit researchers to improve their proposals or even detect in which conditions one solution is more convenient than others. This is the main reason why we propose in this paper a new and free available environment in order to implement and study prefetching techniques efficiently. Our framework is a hybrid implementation that combines both real and simulated parts in order to provide flexibility and accuracy. It reproduces in detail the behavior of web users, proxy severs and original servers. The simulator also includes a module to provide performance results, such as precision (prefetching accuracy), recall, response time, and byte transference.
\end{abstract}

\section{Introduction}

The enormous spread of Internet and consequently, the growth of the World Wide Web due to the popularization of new applications and services, like ebusiness, multimedia contents, e-learning, etc. have implied a dramatic increase in connected users and as a consequence, a tremendous increase in the global traffic, damaging the quality of service (availability, reliability, security) and specially the latency perceived by the users.

Internet latency depends on the number of intermediate points and the amount of traffic crossing them. The connection between two adjacent points is generally a cable line, but data must cross other elements such as modems, Ethernet cards, switchers, routers, etc. All these elements are obstacles. Each point has a separate hardware, different cable speeds, and it even uses different protocols. Therefore, the number of intermediate steps must be minimized and individual bandwidth and speed must also be enhanced in order to improve web architecture performance. The international and global nature of Internet (Internet is a network of networks) makes it very difficult (or sometimes really impossible) to improve system performance working in the networks and their interconnection elements. As a consequence, it is necessary to work in the web architecture and organization using and exporting techniques already learned and widely used in computer architecture to improve its performance.

Those techniques take advantage of the locality properties shown by web objects. In the Web, locality has three different characteristics: temporal, spatial and geographical, allowing us to implement efficiently caching [1,2], prefetching [3,4] and replication techniques $[5,6]$ in order to increase performance.

In this paper we focus on prefetching techniques which make use of the spatial locality that Web objects accesses present. The goal of this technique is to download objects before the user demands them explicitly. The main prefetching mechanisms proposed in the literature are transparent to the user and, consequently, they are necessarily speculative. As any speculative technique, prefetch predictions can fail, i.e. downloading an object that will not be demanded later. That means that prefetching can involve many hazards because if the prediction is not well done, it can pollute the cache, waste bandwidth and overload the original server. This implies that prefetching must be carefully 
applied, by using, for example, idle times in order to avoid performance degradation [7]. Despite these hazards, it has been shown that many prefetching algorithms $[3,4,8,9,10]$ can reduce considerably the perceived latency by users.

The prefetch predictions can be performed by the server, by the proxy, or by the client itself. Some research studies suggest performing the predictions at the server [11] because it is visited by a high number of users and it has enough information about how they visit the site, therefore its predictions can be quite accurate. Some others studies suggest that proxy servers can perform more accurate predictions because their users population is much more homogeneous than in an origin server, and they can also predict crossserver links which can reach about 29\% [12] of the requests. Finally, other authors suggest that predictions must be performed by the client browsers $[4,8,13]$, because it knows better users' preferences. There are also some studies suggesting that the different parts of the web architecture (users, proxies and servers) must collaborate when performing predictions [4]. Although the open literature presents very interesting proposals about prefetching techniques, it still has not been possible to reproduce them (or to propose new ones) using similar environmental conditions in order to compare their main features, advantages and disadvantages. Our main goal in this paper is to propose a simulation environment to check the performance of different prefetching algorithms under similar conditions. Our environment permits the implementation of prefetching algorithms on any part of the Web architecture (user browser, proxy or server), either individually or working together.

The simulation environment (Fig. 1) is composed of three main parts: the server, the client, and the proxy. We have developed a hybrid implementation which combines both real and simulated parts in order to provide the environment flexibility and accuracy.

The remainder of this paper is organized as follows. Section 2 discusses the reasons that motivated us to propose this environment. Section 3 describes the block diagram and components of the proposed tool as well as the performance metrics that it provides. Section 4 illustrates some working examples. Finally, section 5 summarizes some concluding remarks.

\section{Motivation}

Most of the prefetching research studies use different frameworks to check their proposals, therefore the assumptions, conditions, implementations and even performance metrics widely differ among them. This fact implies that performance comparison

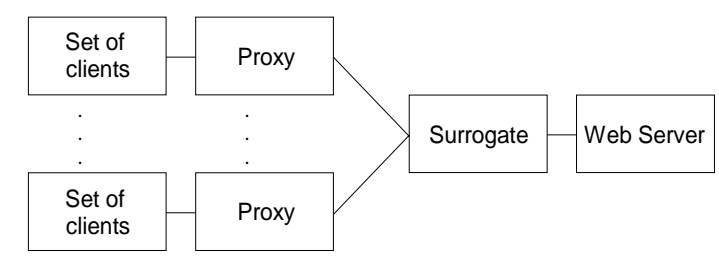

Figure 1. Architecture of the simulation

studies cannot be fairly done. Therefore, it is difficult to distinguish the aspects and conditions in which each technique presents better performance, or its use is more convenient. Some of these studies presented in the open literature analyze the theoretical bounds of performance that prefetching can reach $[12,14]$. The different scenarios only permit to arrive to very general conclusions like, for instance, that performance can boost until $60 \%$ [14] or more [12].

Although there are some simulator environments to check cache replacement algorithms $[15,16]$ as well as network features [17], there is a lack of simulation environments which concentrate on prefetching. This fact, together with the potential performance benefits that prefetching can reach, motivates us to propose this framework which permits to implement and to check the performance of almost any prefetching algorithm.

As mentioned above, our simulator models the environment considering three main parts: the server (including both the server and the surrogate), the client, and the proxy.

- The back end part has both the real web server and the surrogate server. The server is external to our environment and the system can access to it through the surrogate where the prefetching engine is implemented. The surrogate is implemented on a real machine offering fully representativeness.

- The front end has the client component, which represents the user behavior in a prefetch enabled client browser. It is possible to use both real traces and a synthetic workload generator, for each set of users accessing concurrently to the same server.

- The intermediate part, which is between the clients and the surrogate system, models one or more proxy servers, each one can implement prefetching or not.

This organization provides a useful and operative environment since it permits the implementation of prefetching algorithms working in any part of the system (in the browser of the client, in the original server or in the proxy, or even in a collaborative manner) by modifying only few parts of the structure. In addition, the environment provides the appropriate interfaces to communicate the different modules between them, in order to implement easily those 
prefetching algorithms in which the different elements collaborate. On the other hand, our simulator also includes a module to provide performance results, such as precision (prefetching accuracy), recall, response time, and/or transference.

\section{Proposed experimental environment}

As mentioned above, the proposed environment permits to implement prefetching techniques in one or more parts of the system, each one working independently or in a collaborative way. Each part that can perform prefetch has its own entity in the proposed environment: the surrogate proxy, the proxy server and the web client.

\subsection{Surrogate}

A surrogate proxy is an optional element located close to the origin server that generally acts as a cache of the most popular server responses ([18], [19] or accelerator mode in [20]). Usually surrogates are not used to perform prefetching predictions. Although it is equivalent to performing predictions from the server or the surrogate, the last one allows much easier and flexible software implementations. Therefore, we perform our implementation on the surrogate.

The surrogate hints clients about what objects should be prefetched in the same way as Mozilla (from version 1.2 on) admits [21], by means of an optional header offered by the HTTP/1.1 standard [22]. This implementation is based on the one described in [8]. The list of hints that the surrogate proxy piggybacks is generated for each response.

Figure 2 shows the block diagram of the proposed surrogate proxy. Each block represents a functional unit. Some of them are implemented as functions and others as threads, keeping separately all their functionality.

The listener block, which is the simplest one, is implemented as a thread that waits for clients to connect its socket, acting as a concurrent TCP server that listens at a proxy port (generally at 80). By default, there are no restrictions on the number of simultaneous incoming connections. The main goal of this block is to create a new Connection_to_server thread.

The Connection_to_server block, which is the main one, handles the connections. The connection involves a request that is transferred to the web server, which processes it and starts the transference. In this moment, the module first parses the response until the full header is received and then, asks for a prediction to the

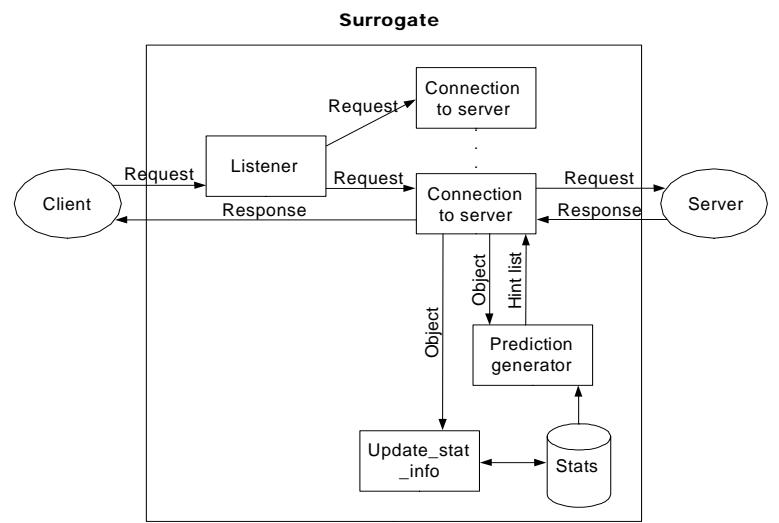

Figure 2. Block diagram of the surrogate

Prediction_ generator block. The prediction consists of a list of hints, whose content depends on the implemented algorithm. This list will be piggybacked on the response following the header, so the client can prefetch the object if the client application considers it appropriate.

When the whole object is received, the listener calls the Update_stat_Info block to notify that event as well as its headers (object size, content type, prefetch or user request, etc). This information is used to update the contents of the statistical database, which is used to generate predictions. The frequency at which statistics are updated depends on the prefetching scheme; e.g., once per hour, once per day, when the server is not too loaded, when the server finishes its response etc. As our aim is to maintain the simulator as flexible as possible, we implemented this module as a function which updates the information or put the fetched object into a queue for processing them later, although using threads to implement it is also possible.

The Prediction_generator block represents the task of generating predictions every time an object is requested. This block generates the prediction list of the objects with the highest probability to be accessed, by taking the statistical database contents as inputs. This list depends on the prefetching algorithm, which can also use external information.

Once described the modules that the surrogate implements, we describe the interface it offers to accommodate new prefetching proposals. Table 1 shows this interface for the different functions that the surrogate part implements. This interface permits to model proposals which do not involve changes in the http 1.1 standard, although it is also possible to model techniques requiring changes in the standard by modifying the corresponding modules. For instance, implementing proposals which require the client to inform the server about the prefeching hits in order to reach more accurate predictions. 
Table 1. Function interface to implement prefetching algorithms

\begin{tabular}{llll} 
Function & Parameters & Result & Description \\
\hline \hline prefetchInit & None & None & $\begin{array}{l}\text { It is called when surrogate is started, and it is used to initialize variables } \\
\text { or to create a thread that updates some information periodically. Server } \\
\text { starts to work when this function returns. }\end{array}$ \\
\hline getHints & $\begin{array}{l}\text { Client IP, Input and } \\
\text { output headers }\end{array}$ & Hints list & $\begin{array}{l}\text { It is called when all response headers are received from the server with a } \\
\text { list of hints of those objects that probably will be requested in the future. }\end{array}$ \\
\hline updateStats & $\begin{array}{l}\text { Client IP, Input and } \\
\text { output headers }\end{array}$ & None & $\begin{array}{l}\text { It is called when all response data has been sent to the client for updating } \\
\text { user access statistics. }\end{array}$ \\
\hline
\end{tabular}

\subsection{Client}

The client part of the system reproduces the behavior of a set of users browsing the WWW with a prefetching enabled web browser. The requests the users generate are obtained from real traces collected by a Squid proxy. This module can be replaced either by other log format or even by any synthetic workload generator.

In order to simulate the behavior of the client, it is important to respect the time intervals between two successive requests. Those times are obtained from timestamp differences observed on logs. As timestamps in logs are made at the end of the transfer time, we need to subtract the duration field to the timestamp on logs to obtain the start time of the transfer. For this purpose, the environment includes a simple "chgtime" script.

According to the real behavior of a prefetching enabled browser like Mozilla, and as several research studies suggest [7,23], the prefetching of objects should be performed only when the user is not downloading objects. Furthermore, those proposals suggest stopping the prefetch connection when a user request rises during the prefetch transference. The client simulator permits to stop the prefetch transference, if wanted, by setting the corresponding parameter (StopPrefetch) in the configuration file. Table 2 shows the parameters that this file includes. This option aborts the prefetch losing the connection; in a future work we plan to study how this connection could be reused to achieve better performance.

The client part has also the ability to introduce latency for each downloaded object offering the time a client waits to get an object. There are three parameters in the simulator affecting the response time of an object: the connection time, the server, processing time and the available bandwidth.

These parameters are not enough to model closely a real network. To this end, an external delay generator can be included in the simulator, like the ns 2 proposed in [26]. By using a generator it is possible to simulate scenarios having any particular kind of clients; e.g., systems with wireless clients losing a lot of packets and/or cable users with a high bandwidth. Analogously, it is possible to model different network conditions on the server; e.g., congestion and packet loss.

Table 2. Configuration file. Parameters and description

\begin{tabular}{ll} 
Parameter & Description \\
\hline \hline StopPrefetch & $\begin{array}{l}\text { Stop or not prefetch requests when a new } \\
\text { user request is performed }\end{array}$ \\
\hline HintsOfPrefetch & $\begin{array}{l}\text { The moment the client includes the } \\
\text { received hints attached to the prefetched } \\
\text { objects in the hint list: 0, when received; } 1, \\
\text { when prefetched object is accesed }\end{array}$ \\
\hline MaxLongHintL & $\begin{array}{l}\text { Maximum length of the list of hints (for the } \\
\text { client) }\end{array}$ \\
\hline Bandwidth & $\begin{array}{l}\text { Max number of bytes a user can receive per } \\
1 \text { second }\end{array}$ \\
\hline EnablePrefetch & $\begin{array}{l}\text { Type of prefetching hints: 0, no prefetch; 1, } \\
\text { prefetch when hints received; and 2, only } \\
\text { when idle }\end{array}$ \\
\hline ConnectDelay & $\begin{array}{l}\text { How many ms a new connection to the } \\
\text { server is delayed }\end{array}$ \\
\hline ServerDelay & $\begin{array}{l}\text { How many ms a new request to the server } \\
\text { is delayed }\end{array}$ \\
\hline Connections & $\begin{array}{l}\text { Max number of connections a client can do } \\
\text { simultaneously to server }\end{array}$ \\
\hline http11 & \begin{tabular}{l} 
HTTP version (1.1 or 1.0) \\
\hline ClearList
\end{tabular} \\
$\begin{array}{l}\text { It tells the simulator whether to clear the } \\
\text { hint list when a new user connection arrives } \\
\text { after an idle period }\end{array}$ \\
\hline
\end{tabular}

When simulating cache clients, the simulator only stores the object references in order to avoid that the object is fetched from the server later. Note that, for our purposes, it is not necessary to store the content of each object. However, as explained below, this must 
be considered when working in the client part of the proxy cache.

Once the hints attached to the prefectched objects are received, they must be included into the prefetching list. The parameter shown in Table 2 as HintsOfPrefetched, states the moment at which they are included. There are two alternatives: i) when received, and ii) when accessed the prefetched object.

Figure 3 shows the block diagram of the client simulator. As observed, the client part communicates only with the Squid traces, the log file (for analyzing later the results achieved by prefetching), and the server. The latter, depending on the modeled system, could be replaced by one or more proxies.

There are two data stores inside the client: the Prefetch_List and the Fetched_Objects. The Prefetch_List contains the list of objects that the server hints the client to prefetch. These hints have been piggybacked with the requested object by the user, and accumulated for each burst of user requests. When there is a new user request after an idle period, the remaining hints in the list are removed. If one does not want to remove the hints, the corresponding variable (ClearList) in the configuration file must be set to zero. The Fetched_Objects data store keeps all the fetched objects and, if they are prefetched, stores also whether the user has accessed them later or not. This information can be used to simulate an infinite cache in the client, because objects in this data store are never fetched or prefetched. Of course, this behavior can be deactivated in the simulator by means of the corresponding parameter (EnableCache) in the configuration file.

The Read_Traces block reads the users requests from a Squid log. This module reads each access and inserts it in a different FIFO queue for every client. Each insertion is timed basing on timestamps of the Squid log file to match the access pattern of the original user. It works in a similar way the HTTP Blast does [24], (it is a tool that reads a trace file with URLs and submit them to a proxy cache) but integrated into the client simulator. This block is implemented as an independent thread connected to the rest of the system through FIFO queues. As it is independent, it can be easily replaced by a synthetic workload generator to feed the environment with the desired number of clients and requests. The way of managing multiple clients is by means of multiple processes (one per client) that work independently to request objects to the server.

The Prefetch Manager block reads requests from the Read_Traces queue and asks for the object if it has not been prefetched yet (by checking the Fetched_Objects data store). In order to request files to

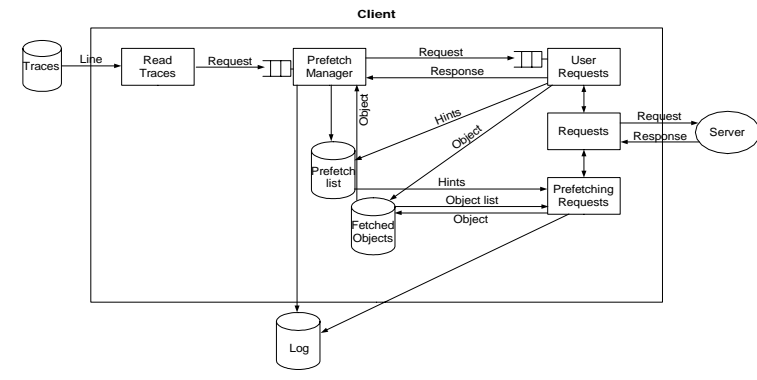

Figure 3. Block diagram of the client part

the server, the Prefetch_Manager has many User_Request threads (the connections parameter of the configuration file) created and waiting for a new request. When the object is received, if its URL is in the Prefetch_List, it is removed. Then, that URL is inserted into Fetched_Objects data store in order to avoid prefetching the object later.

If the Read Traces queue is empty and the prefetch parameter is set to Only_when_idle, the Prefetch_Manager allows the prefetching until a new user request rises. When a request is inserted into the queue, the Prefetch_Manager indicates that the prefetched downloads must be cancelled and then the Hint_List data store is cleared. Both behaviors are configurable by using the EnablePrefetch and ClearList parameters, respectively.

The User Requests block receives requests from the Prefetch Manager and redirects them to the Requests block, reusing the socket when using the HTTP 1.1 version. When an object is received, this block inserts the prefetching hints piggybacked with the object into the Prefetch_List data store, and the URL of the object into the Fetched_Objects data store in order to avoid the prefetching of the object later. If it already exists, the reference of that object is removed from the Prefetch_List.

The Request block is a common interface (used both by user and prefetch requests) to communicate with the web server (or the proxy). This block handles at low level the communication sockets with the possibility of reusing connections if the client is configured to use HTTP/1.1 persistent connections [22].

When the simulator is running, it writes a log file that gathers information about each request to the web server as well as the client request hits to the prefetched object cache. By analyzing the log file, the most relevant performance metrics (recall, precision, bandwidth overhead, etc) can be obtained. To this end, we implemented the results generator program, which obtains the following parameters: the amount of user 
accessed objects, cache hits, prefetch hits, prefetched objects, objects requested to the server, the sum of the response time of objects, total bytes transferred, precision of prefetch and recall.

Note that the process of metrics generation is done at post-simulation time from the Log generated file.

\subsection{Proxy server}

Doing prefetch from the proxy server has many advantages. First, it allows users of non-prefetching browsers (the majority of them at this moment, like Internet Explorer) to benefit from server prefetch hints, analogously to the way we discussed above with respect to the surrogate. Second, it has information from more sources (the clients and the servers), so it is the one that can make more complex and precise predictions about how many and which objects should be prefetched. For example, we could decide to prefetch only those objects cached at the proxy server (as proposed in [3]) or restrict the amount of bandwidth overhead tolerated for prefetching purposes. As predictions are more accurate, it uses less bandwidth and also it involves fewer overloads in the server.

When the different parts of the system support prefetch, many different behaviors can be chosen. For example, the proxy server could receive hints from the server and after processing them, it could start a prefetch connection to cache the selected objects. Then, the proxy could redirect the received hints to the client. These hints could be also removed or even updated (omitting or adding new hints) by applying its own proxy prediction algorithm, since the proxy has a better knowledge of its users than the server has. In addition, the proxy can observe cross-server links.

The structure of the prefetching proxy server can be seen as a combination of a surrogate and a client. On one hand, the proxy waits for connections and performs predictions like the surrogate; on the other hand, the proxy requests files to the server and processes hints like the client. From this point of view, only few changes should be performed, i) the objects must be stored, and ii) requests to the server are generated from client requests (instead of from log traces as done in the client part).

By having all these three parts (surrogate, client, and proxy) we can: i) check any proposal already appeared in the literature and ii) create novels test environments which inter-relate the three parts of the global system. In addition, the proxy side can be split in more parts to check the performance of new organizations; e.g. prefetching between proxies.

Simulations can be run by just setting up one set of clients and one server, which is the simplest system
Table 3. Example of performance results

\begin{tabular}{rccc}
\multicolumn{1}{c}{ Prefetch value } & $\mathbf{0}$ & $\mathbf{1}$ & $\mathbf{2}$ \\
\hline \hline User requests & 11363 & 11363 & 11363 \\
\hline Avg. resp. time (ms) & 330.06 & 290.17 & 247.87 \\
\hline \% reduced & N/A & $12.1 \%$ & $24.9 \%$ \\
\hline Total transfer (KB) & 32187 & 43229 & 50003 \\
\hline \% increased & N/A & $34.3 \%$ & $55.3 \%$ \\
\hline Precision & N/A & $46.1 \%$ & $44.4 \%$ \\
\hline Recall & N/A & $15.7 \%$ & $22.0 \%$ \\
\hline
\end{tabular}

that can be modeled. The system can become more and more complex to represent more realistic scenarios.

To run simulations, the three parts of the modeled system can be placed in a single computer or placed distributed among computers of the same or different LANs. The number of clients that a computer machine can simulate depends on the CPU requirements of the client prefetching algorithm.

\section{Implementation example}

As an example of how a prefetch technique can be implemented in our environment, we use the proposed by Padmanabhan and Mogul [8]. We omit some implementation details since the goal of this paper is only to present the simulation environment.

This proposal takes into account a set of clients and the server. The server implements a prefetching algorithm similar to the proposed by Griffioen and Appleton [25], which uses previous client-requests to generate the prediction list piggybacked on the response. Then, the client receives the list, and uses it when it is idle to ask the server for the hinted objects. When a user generates a real request, the prefetch is stopped.

The behavior pattern of the users was taken from logs collected by a Squid proxy of the Polytechnic University of Valencia. We selected those requests accessing to a popular news web server, which was mirrored to avoid external interferences. The response time parameters $(50 \mathrm{~ms}$ ConnectionDelay and $150 \mathrm{~ms}$ ServerDelay) were obtained experimentally by timing connections to the real server. The others parameters were taken according to the HTTP/1.1 standard and Mozilla implementation:

- A maximum of two connections to the server per client.

- The system only prefetchs when there are no users requests in course (including the stop of prefetch 
connections when the user makes a new request).

- Hints list (per client) is cleared after an idle period.

- The hints piggybacked with the prefetched objects are only processed when the container object is accessed.

According to the interface presented with the surrogate, accommodating the selected algorithm in the proposed environment is limited to the implementation of the next three functions:

We create a thread in the prefetchInit to update statistical information of user requests (probability of next access for each file) every ten seconds.

The updateStats function marks the object being accessed as a possible successor of previous objects accessed by the user.

The getHints function obtains a list of those objects having a probability equal or higher than $40 \%$ of being accessed within the next 4 accesses as suggested in [8].

Table 3 illustrates the results obtained by the environment for the selected prefetching algorithm when non-prefetching and with two different prefetching methods. The results were obtained considering an initial empty cache and a small trace, so that little past information can be used for prediction. However, we want to remark that it is used only with illustrative purposes.

Figure 4 profiles a little set of requests from three points of view: the web server, the surrogate, and the client logs. This profile shows the dynamic behavior of the requests and can help us to debug the prefetching algorithms implemented. URLs are cut in the example due to their length. Some other characteristics that can be observed are:

- The IP address of the six requests that the web server receives is the corresponding to the surrogate. So the server sees all the requests as if they come from the surrogate. Timestamps are omitted.

- The surrogate distinguishes between user and prefetch requests through the $\mathrm{X}-\mathrm{moz}$ header.

- The first column of the client is the timestamp of the request and the second one the request type (U: user, P: prefetch).

As the surrogate submits hints with the object, the size of the response that the user receives (third column of the client log) differs from that submitted by the server (sixth column of the server).

\section{Conclusions}

Due to the large amount of research concentrating on prefetching, their results are difficult to compare because of their different conditions, assumptions, and implementation considered among the studies.

In this work we have proposed an experimental framework to implement prefetching algorithms. Thus, as algorithms are implemented under the same environment, performance comparison studies can be fairly done. In addition, the environment offers some

\begin{tabular}{|c|c|c|c|c|}
\hline \multicolumn{5}{|c|}{ Web Server } \\
\hline $158.42 .58 .137-$ & [timestamp] & "GET / HTTP/1.1" & 20072460 & "- \\
\hline $158.42 .58 .137-$ & [timestamp] & "GET .../relojsandoz2003_2.swf HTTP/1.1" & $200 \quad 6799$ & "-" " " \\
\hline $158.42 .58 .137-$ & [timestamp] & "GET .../david_ferrer030505.jpg HTTP/1.1" & 1138 & "-" " " " \\
\hline $158.42 .58 .137-$ & [timestamp] & "GET .../posicionhome.css HTTP/1.1" & 851 & "-" " " \\
\hline $158.42 .58 .137-$ & [timestamp] & "GET .../gerard@30402.jpg HTTP/1.1" & 1124 & "-" " " " \\
\hline \multicolumn{5}{|c|}{ Surrogate } \\
\hline \multirow{2}{*}{\multicolumn{5}{|c|}{  }} \\
\hline & $\mathrm{TP} / 1.12000$ & & & \\
\hline \multirow{2}{*}{\multicolumn{5}{|c|}{ Header 7-8-->Link: <http://WWW.marca.com/.../relojsandoz2003_2. swf>; rel=prefe }} \\
\hline & & WWW.marca.com/.../david_ferrer $030505 . j p g$ & ; rel=pref & tch \\
\hline \multirow{2}{*}{\multicolumn{5}{|c|}{ Header 7-0<--GET /.../relojsandoz2003_2.swf HTTP/1.1 }} \\
\hline & & ( & & \\
\hline \multicolumn{5}{|c|}{ Header 7-0-->HTTP/1.1 200 OK } \\
\hline \multicolumn{5}{|c|}{ Header 7-0<--GET /.../david_ferrer030505.jpg HTTP/1.1 } \\
\hline \multicolumn{5}{|c|}{ Header 7-1<--X-moz: prefetch } \\
\hline \multicolumn{5}{|c|}{ Header 7-0-->HTTP/1.1 200 OK } \\
\hline \multirow{2}{*}{\multicolumn{5}{|c|}{ Header $9-0<--$ GET /.../posicionhome.css HTTP/1.1 }} \\
\hline & & & & \\
\hline \multicolumn{5}{|c|}{ Header 9-8-->Link: <http://WwW. } \\
\hline \multirow{2}{*}{\multicolumn{5}{|c|}{$\begin{array}{l}\text { Header 9-9-->Link: <http://WWW. marca.com/../gerard030402.jpg>; rel=prefetch } \\
\text { Header 9-0<--GET/.../gerard030402.jpg HTTP/1.1 }\end{array}$}} \\
\hline & & & & \\
\hline \multirow{2}{*}{\multicolumn{5}{|c|}{$\begin{array}{l}\text { Header } 9-1<--X-\text { moz: prefetch } \\
\text { Header } 9-0-->\text { HTTP/1, } 1200 \text { OK }\end{array}$}} \\
\hline & & & & \\
\hline \multicolumn{5}{|c|}{ Header 9-0-->HTTP/1.1 200 OK } \\
\hline \multicolumn{5}{|c|}{$1056985840.876 \quad$ U $73254 \quad 269 \quad 127.42 .92 .155$ http://WWW.marca.com/ } \\
\hline \multicolumn{5}{|c|}{127.42 .92 .155 http://wWW.marca.com/ } \\
\hline \multicolumn{2}{|c|}{$1056985841.248 \quad P \quad 1827 \quad 160$} & 127.42 .92 .155 http://wWW.marca.com/ & /david_fer & er $030505 . j p g$ \\
\hline & 127.42 .92 .155 http://wWw. marca.com/ & /posicionh & me.css \\
\hline \multicolumn{2}{|c|}{$\begin{array}{llll}1056985841.448 & \text { U } & 1391 & 220 \\
1056985841.668 & P & 1843 & 220\end{array}$} & 127.42.92.155 http://WWW.marca.com/... & /gerard030 & 02. jpg \\
\hline
\end{tabular}


interfaces to ease the implementation and test of new algorithms. Therefore, algorithms can be accommodated in the general environment by modifying only few modules, independently of the entity implementing prefetch; i.e., the surrogate or the client.

The tool can be freely accessed in http://remo.disca.upv.es/prefetchsimulator. Both the surrogate and the client part have been tested and polished, and we are now polishing minor implementation details in the proxy. We hope that the proposed tool can help researchers in their job.

In summary, our proposal offers an experimental framework for testing a huge variety of web architecture related aspects. Among them, the main contributions of the proposed environment reside in the following capabilities:

1.Quantifying how the prefetch requests can interfere in the performance of the user requests. Even more, we can quantify both self-interference and the crossinterference [23]. This fact is possible because our environment includes the entire system.

2.Standardizing the evaluation of prefetching techniques for comparison purposes.

3.Creating novel prefetching architectures that interrelate the three parts of the global system. Therefore, we can implement prefetching algorithms in any point of the system as well as to check their impact on the overall system performance.

4.Classifying for each user profile which of the prefetching technique offers the best performance server, the surrogate and thef cient assumptions and conditions.

\section{References}

[1] S. Jin, A. Bestavros, "Popularity-aware GreedyDualSize web proxy caching algorithms," Proc. of the Conference on Distributed Computing Systems, Taipei, Taiwan, 2000.

[2] C. Aggarwal, J. L. Wolf, and P. S. Yu, "Caching on the World Wide Web," IEEE Transactions on Knowledge and Data Engineering, vol.11, no.1, pp. 94-107, 1999.

[3] L. Fan, P. Cao, and Q. Jacobson, "Web prefetching between low-bandwidth clients and proxies: potential and performance," Proc. of the ACM SIGMETRICS Conference on Measurement and Modeling of Computer Systems, Atlanta, USA, 1999.

[4] E. P. Markatos, C. E. Chronaki, "A Top-10 Approach to Prefetching the Web," Proc. of the INET' 98, Geneva, Switzerland, 1998.

[5] A. Iyengar, E. Nahum, A. Shaikh, R. Tewari, "Enhancing Web Performance," Proc. of the IFIP World Computer Congress, Montreal, Canada, 2002.

[6] K. L. Johnson, J. F. Carr, M. S. Day, M. F. Kaashoek, "The Measured Performance of Content Distribution Networks," Proc. of the $5^{\text {th }}$ International Web Caching and Content Delivery Workshop, Lisbon, Portugal, 2000.
[7] M. Crovella, P. Barford, "The network effects of prefetching," Proc. of the IEEE INFOCOM'98 Conference, San Francisco, USA, 1998.

[8] V. N. Padmanabhan, J. C. Mogul, "Using Predictive Prefetching to Improve World Wide Web Latency,” Proc. of the ACM SIGCOMM '96 Conference, Palo Alto, USA, 1996. [9] X. Chen, X. Zhang, "Popularity-Based PPM: An effective web prefetching technique for high accuracy and low storage," Proc. of the 2002 International Conference on Parallel Processing, Vancouver, Canada, 2002.

[10] A. Bestavros, "Using speculation to reduce server load and service time on the WWW," Proc. of the 4th ACM International Conference on Information and Knowledge Management, Baltimore, USA, 1995.

[11] C. R. Cunha, C. F. B. Jaccoud, "Determining WWW user's next access and its applications to pre-fetching," Proc. of the $2^{\text {nd }}$ International Symposium on Computers and Communication, Alexandria, Egypt, 1997.

[12] D. Duchamp, "Prefetching Hyperlinks," Proc. of the $2^{\text {nd }}$ USENIX Symposium on Internet Technologies and Systems, Boulder, USA, 1999.

[13] E. Cohen, H. Kaplan, "Prefetching the means for document transfer: A new approach for reducing web latency," Proc. of the IEEE INFOCOM'00 Conference, TelAviv, Israel, 2000.

[14] T. M. Kroeger, D. D. E. Long, J. C. Mogul, "Exploring the bounds of web latency reduction from caching and prefetching," Proc. of the USENIX Symposium on Internet Technologies and Systems. Monterey, California, USA, 1997.

[15] L. G. Cárdenas, J. Sahuquillo, A. Pont, J. A. Gil, “The Multikey web cache simulator: A platform for designing proxy cache management techniques," Proc. of the $12^{\text {th }}$ Euromicro Conference on Parallel, Distributed and Network based Processing, La Coruña, Spain, 2004.

[16] P. Cao, "Wisconsin web cache simulator," http://www.cs.wisc.edu/ cao/

[17] B. D. Davison, "NCS: Network and cache simulator an introduction,” Technical Report DCS-TR-444, Department of Computer Science, Rutgers University, USA, 2001.

[18] Network Working Group, "Internet web replication and caching taxonomy,” RFC 3040, 2001.

[19] A. Vahdat, E. Belani, P. Eastham, C. Yoshikawa, T. Anderson, D. Culler, M. Dahlin, "WebOS: Operating system services for wide area applications," Proc. of the $7^{\text {th }}$ Symposium on High Performance Distributed Computing, Chicago, USA, 1998.

[20] O. Pearson, "Squid user's guide," http://squiddocs.sourceforge.net/latest/book-full.html\#AEN2416.

[21] D. Fisher, G. Saksena, "Link prefetching in Mozilla: A server-driven approach," Proc. of the $8^{\text {th }}$ International Workshop on Web Content Caching and Distribution, New York, USA, 2003.

[22] R. Fielding, J. Gettys, J. Mogul et al, "Hipertext transfer protocol - HTTP/1.1,” 1998.

[23] R. Kokku, P. Yalagandula, A. Venkataramani, M. Dahlin, "NPS: A Non-Interfering Deployable Web Prefetching System," Proc. of the USENIX Symposium on Internet Technologies and Systems, San Antonio, USA, 2003. 
[24] J. Vöckler, "HTTP Blast," http://www.cache.dfn.de/DFN-Cache/Development/blast.htm [25] J. Griffioen, R. Appleton, "Reducing File System Latency using a Predictive Approach," Proc. of the USENIX
Summer 1994 Conference, Santa Fe, USA, 1994.

[26] UCB/LBNL/VINT, "Network simulator ns (version 2)," http://www.isi.edu/nsnam/ns 